\title{
Reutilización de yeso recuperado de construcciones: un estudio basado en requisitos de aptitud de normas argentinas y chilenas
}

\author{
Reuse of recovered construction gypsum plaster: a study based on aptitude requirements of \\ argentine and Chilean standards
}

\author{
Hugo Begliardo (Autor Principal y Autor Oficial de Contacto). \\ hugo.begliardo@frra.utn.edu.ar \\ Universidad Tecnológica Nacional, Facultad Regional \\ Rafaela, Departamento Ingeniería Civil, Argentina \\ Fono 543492 422880, Fax 543492432710 \\ Acuña 44, CP S2300, Rafaela, Argentina
}

\section{Mirta Sánchez}

Universidad Tecnológica Nacional, Argentina

mirta.sanchez@frra.utn.edu.ar

\section{Resumen}

El incremento de la actividad de la construcción, acompañando la globalización de la economía, dio lugar a un gran crecimiento en la generación de residuos de construcción y demolición. El yeso forma parte del problema que acarrea su disposición final, puesto que sus residuos suelen tener por destino los Rellenos Sanitarios o vertederos, sin que se aproveche su potencialidad de reciclado. El depósito sin control puede provocar, entre otros efectos, contaminación del lecho freático y generación de gases tóxicos. Estas razones, sumadas al alto costo de su disposición final, motivaron a que muchos países dictasen directivas sobre resguardos en el vertido, procurando evitar impactos medioambientales negativos y promoviendo su reaprovechamiento. En consonancia con lo último, el objeto de este trabajo es presentar los resultados de estudios y ensayos de laboratorio realizados sobre mezclas con residuos de yeso, a fin de analizar la viabilidad de su reutilización, a partir de la evaluación del ajuste a determinados requisitos establecidos en las normas argentina IRAM 1607:70 y chilena NCh 143.Of1999. Los residuos, debidamente procesados, fueron incorporados en diferentes porcentajes en una matriz de yeso comercial. Los resultados hallados demuestran que es factible su reaprovechamiento, con los consiguientes beneficios medioambientales que conlleva.

Keywords: Recycled gypsum; Environment; Standards.

\section{Introducción}

El yeso es un noble material de construcción cuyo uso se remonta a tiempos muy antiguos, cuando durante la revolución neolítica se alcanzó el nivel tecnológico necesario para dominar el fuego a bajas temperaturas (Villanueva, 2005). Se tienen registros de su empleo en el IX milenio a.C. en Anatolia, Turquía, y en el VI milenio a.C. en Jericó, Cisjordania. En España tuvo amplia difusión a partir de la conquista árabe (Villanueva, 2004; Asociación Técnica y Empresarial del Yeso [ATEDY], 2006); tras ello dicha nación influyó significativamente en el uso y diferentes aplicaciones que se dio al yeso en América Latina. Su nobleza radica en un conjunto de propiedades y ventajas que lo destacan entre otros materiales: es fácilmente moldeable, liviano y estético; buen aislante térmico y acústico; adhiere excelentemente a materiales porosos y su fraguado es rápido, aunque regulable con aditivos; resiste al fuego, entre otras razones debido a su porosidad y alto punto de fusión del $\mathrm{CaSO}_{4}$; el costo para su obtención es relativamente bajo (Gomá, 1979).

\author{
M. Cecilia Panigatti \\ Universidad Tecnológica Nacional, Argentina \\ maria.panigatti@frra.utn.edu.ar \\ Sofía Garrappa \\ Universidad Tecnológica Nacional, Argentina \\ sofia.garrappa@frra.utn.edu.ar
}

\author{
Código: 0162 \\ Fecha de Aceptación: 1 de diciembre de 2013
}

\section{Abstract}

The increase in construction activity, accompanying the globalization of the economy has led to a huge growth in the generation of construction and demolition waste. Gypsum is part of the problem that leads the final disposal of them, since plaster waste usually have final destination in landfills, without take advantage of its recycling potential. The uncontrolled deposit of residual gypsum plaster can cause, among other effects, contamination of ground water and the generation of toxic gases. These and other reasons, coupled with the high cost of disposal, have prompted that many countries began to issue directives on safeguards in the disposal, avoiding negative environmental impacts and promoting its reuse. Aligned with this, the aim of this paper is to present the results of studies and laboratory tests of mixtures with gypsum waste in order to analyze the feasibility of it reuse, assessing their adjustment to certain requirements established in argentine IRAM 1607:70 and chilean NCh 143.Of1999 standards. Wastes, properly processed, were incorporated in different percentages in a commercial gypsum matrix. Results shows that it is feasible to reuse, with consequent environmental benefits.

Se puede afirmar que el yeso es un material tradicional y nuevo a la vez, de uso milenario y muy actual en sus aplicaciones, por cuanto la investigación y nuevas técnicas de fabricación van rejuveneciéndolo con el tiempo al punto de que muchos de sus múltiples derivados se pueden entender como no tradicionales (ATEDY, 2006). Su versatilidad lo distingue, puesto que con el agregado de aditivos y/o adiciones, pueden lograrse con él productos con disímiles características y de variadas aplicaciones, bien sea para ser utilizados en condiciones húmedas, bajo forma de pastas o morteros (ejecución de revestimientos de paredes y cielorrasos), o en condiciones secas mediante prefabricados (placas laminadas, ladrillos, molduras)

La materia prima natural para su obtención es, básicamente, la roca sedimentaria de origen evaporítico denominada "piedra de yeso". Esta es la génesis más común de las cuencas yesíferas en América Latina, desde México hasta Argentina (Ponce y Torres Duggam, 2005). Esta roca está compuesta, fundamentalmente, por sulfato de calcio dihidratado 
$\left(\mathrm{CaSO}_{4} \cdot 2 \mathrm{H}_{2} \mathrm{O}\right)$, acompañado en mayor o menor grado de algunas impurezas, tales como arcillas, arenas, otras sales $y$, especialmente, anhidrita (Sanz, 2009).

La calcinación de la piedra de yeso a diferentes temperaturas lo transforma en diversas fases y estados alotrópicos. El yeso comercial en polvo utilizado en la construcción es un aglomerante bajo la forma de hemihidrato $\left(\mathrm{CaSO}_{4} \cdot \frac{1}{2} \mathrm{H}_{2} \mathrm{O}\right)$, el cual se obtiene deshidratando a temperaturas relativamente bajas $\left(100-130^{\circ} \mathrm{C}\right)$ al sulfato de calcio dihidratado (Gomá, 1979). Con el agregado de agua recupera las moléculas perdidas durante el proceso de calentamiento y se vuelve a transformar en dihidrato, más poroso y menos denso que el original (González Madariaga, 2005).

Otra fuente de generación de yeso, químicamente idéntico al yeso natural, es su obtención a partir de diversos procesos industriales, entre ellos por desulfuración de gases de combustión. Se lo conoce como yeso sintético o químico. Actualmente se está transformando en un importante sustituto del yeso natural en la fabricación de paneles, la producción de cemento y aplicaciones agrícolas. Solo en Estados Unidos su aporte al suministro de yeso nacional pasó del $24 \%$ en 2002 al 54\% en 2012 (U.S. Geological Survey [USGS], 2003, 2013).

La producción mundial de yeso natural en 2012 fue de 150 millones de toneladas (Mt). China, España y EEUU se encuentran entre los mayores productores. En Sudamérica se destacan Brasil (2,8 Mt), que cuenta con una de las mayores reservas mundiales, y Argentina (1,2 Mt) (Ponce y Torres, 2005; USGS, 2013). La mayor parte de la producción mundial tiene por destino la industria de la construcción. Dentro de este campo, en los últimos años se percibe el enorme crecimiento que ha tenido en nuestros países la aplicación del yeso en condiciones secas (placas para tabiques y cielorrasos, bloques) tendiendo a desplazar la tradicional, de aplicación artesanal, en revestimientos de muros y cielorrasos. Sin embargo, este hecho no es nuevo en el hemisferio norte: los tableros o placas de este material son el principal componente en la construcción de paredes. Anualmente se producen en el mundo $80 \mathrm{Mt}$ de placas de yeso, el $85 \%$ de ello corresponde a EEUU, Europa y Japón (Gypsum Recycling International [GRI], 2013).

El crecimiento de la actividad de la construcción, acompañando la globalización de la economía, ha dado lugar a un gran aumento en la generación de residuos de construcción y demolición (RCD). El yeso forma parte del problema que acarrea la disposición final de los mismos, puesto que el descarte por sobrante, fraguado, caídas o rechazo de demoliciones suele tener por destino final los Rellenos Sanitarios (RS) o vertederos. Anualmente $15 \mathrm{Mt}$ (40.000 t/día) de productos que lo contienen se vuelcan a vertederos (GRI, 2013; Ahmed et al., 2011a), de las cuales $4 \mathrm{Mt}$ corresponden a EEUU. Solo una pequeña cantidad es reciclada en ese país, básicamente con propósitos agrícolas y en la manufactura de nuevos paneles de yeso (USGS, 2013).

El vuelco de residuos de yeso en vertederos no controlados altera la alcalinidad del suelo y contamina el lecho freático (Farías y Pimentel, 2010); su disposición en RS debe evitar el contacto con residuos orgánicos y la exposición a la lluvia en ambiente anaeróbico a fin de no generar la emisión de sulfuro de hidrógeno (Lund-Nielsen, 2010). Estas razones, entre otras, sumado al alto costo de la disposición, han dado lugar a que muchos países, entre ellos los comprendidos en la Unión Europea (UE), hayan comenzado a dictar disposiciones en relación al vertido de RCD procurando evitar impactos medioambientales negativos (Unión Europea, 1999, 2003; Lund-Nielsen, 2010). En dicha dirección se alinean las leyes llamadas de "basura cero", vinculadas al reaprovechamiento, reutilización o reciclado de residuos.

Existen actualmente técnicas que permiten reciclar el 100\% de los residuos procedentes de paneles laminados. El polvo de yeso reciclado puede reemplazar hasta un $25 \%$ del yeso natural, o bien del sintético, en la producción de nuevos tableros (GRI, 2013). En el primero de los casos, ello contribuye a la preservación de un recurso minero, fundamentalmente en los países no productores de yeso químico. Las posibilidades de reciclado de yeso también encuentran mercados potenciales de aplicación en la estabilización de taludes y mejoramiento de suelos (Ahmed et al., 2011a; Ahmed et al., 2011b; Ahmed \& Ugai, 2011), absorción de grasas, tratamiento de aguas y secado de lodos (USGS, 2013).

En consonancia con lo anterior, el objeto de este trabajo es presentar los resultados de estudios y ensayos de laboratorio realizados con residuos de yeso de obras aplicado en condiciones húmedas (revestimientos de paredes y cielorrasos) y secas (placas laminadas), incorporados en distintas proporciones a yeso comercial de construcción, como parte de una investigación que ha tenido por finalidad analizar la viabilidad de su reutilización en la misma disciplina, a partir de la evaluación de su ajuste a determinados requisitos establecidos en las normas argentina IRAM 1607:70 (IRAM $1607,1970)$ y chilena NCh 143.Of.1999 (NCh 143,1999). La primera establece las características que deberán tener dos tipos de yesos para construcción, el llamado yeso cocido blanco o tipo A, apto para enlucidos (revoques finos), y el yeso cocido gris o tipo $B$, para revestimientos de primera mano (revoques gruesos). La restante establece las condiciones que debe cumplir el yeso calcinado en Chile.

Se practicaron determinaciones físico-químicas y confeccionaron probetas que fueron sometidas a ensayos físicos, conforme a normas argentinas, chilenas y procedimientos propios. Los resultados que se presentan ponen énfasis en evaluar su encuadre dentro de las normas citadas, proveyendo valores indicativos que determinan el rango dentro del cual es factible reutilizar el material reciclado en su fin primigenio. Complementariamente, se proveen datos obtenidos sobre el módulo de elasticidad, densidad, porosidad y la relación tracción/compresión diametral del material resultante de cada mezcla.

\section{Materiales y métodos}

Los residuos de placas de yeso con cobertura de cartón (YS) recogidos de obras civiles pertenecieron a una misma marca comercial, a fin de homogeneizar la calidad del material para los estudios. A los trozos recuperados se los sometió a un proceso manual de separación gruesa de impurezas, tras lo cual se les extrajo la cobertura, previa inmersión en agua, para luego pasarlos a un tratamiento de secado, trituración, molienda y posterior cribado por el tamiz $N^{\circ} 16$ (IRAM 1,18 $\mathrm{mm}$ ). Para los yesos tipo A es requisito físico que el $100 \%$ del material pase a través del mismo. Tras ello, se llevó el producto a distintas temperaturas de secado de crecimiento escalonado $\left(100,110,120,130\right.$ y $\left.140^{\circ} \mathrm{C}\right)$. Al material recuperado de las obras en las que el yeso se aplicó en condiciones húmedas (YH), debido a rechazos por fraguado, caídas o sobrantes, se le dio en 
laboratorio a un tratamiento similar al dado a los residuos YS (Figura 1).

Por cada temperatura de secado se elaboraron tres tipos de mezclas, tanto para las compuestas con YS como con YH. Cada una de ellas estuvo conformada por la inclusión de yeso recuperado, en un 10, 20 y $30 \%$ del peso total, en una matriz de yeso cocido blanco para construcción. Este, tomado como patrón de referencia (YP), perteneció a una de las marcas de mayor demanda en el mercado argentino la que, sin embargo, no cuenta con el sello optativo de certificación IRAM en sus envases. Las treinta mezclas que integraron lo estudiado, además de la del YP, se identifican en la Tabla 1.

Figura 1. Izq.: Yeso recuperado de placas (YS). Der.: Yeso recuperado de aplicaciones húmedas (YH). Fuente: Elaboración Propia, 2013.
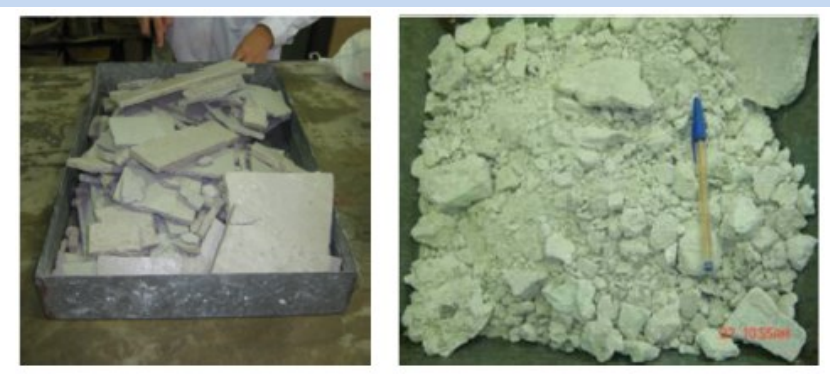

Tabla 1. Mezclas estudiadas. Fuente: Elaboración Propia, 2013.

\begin{tabular}{|c|c|c|c|}
\hline \multicolumn{2}{|c|}{ Designación } & $\begin{array}{c}\text { Residuo de } \\
\text { yeso } \\
\text { incorporado }\end{array}$ & $\begin{array}{c}\text { Temperatura } \\
\text { de secado del } \\
\text { residuo }\end{array}$ \\
\hline \multicolumn{2}{|c|}{ YP-0 (Yeso Patrón) } & $0 \%$ & - \\
\hline $\begin{array}{c}\text { Mezclas con } \\
\text { YS }\end{array}$ & $\begin{array}{c}\text { Mezclas con } \\
\text { YH }\end{array}$ & & \\
\hline YS $10-100$ & YH 10-100 & $10 \%$ & \multirow{3}{*}{$100 \pm 1^{\circ} \mathrm{C}$} \\
\hline YS 20-100 & YH 20-100 & $20 \%$ & \\
\hline YS 30-100 & YH 30-100 & $30 \%$ & \\
\hline YS $10-110$ & YH 10-110 & $10 \%$ & \multirow{3}{*}{$110 \pm 1^{\circ} \mathrm{C}$} \\
\hline YS 20-110 & YH 20-110 & $20 \%$ & \\
\hline YS $30-110$ & YH 30-110 & $30 \%$ & \\
\hline YS $10-120$ & YH 10-120 & $10 \%$ & \multirow{3}{*}{$120 \pm 1^{\circ} \mathrm{C}$} \\
\hline YS 20-120 & YH 20-120 & $20 \%$ & \\
\hline YS 30-120 & YH 30-120 & $30 \%$ & \\
\hline YS $10-130$ & YH 10-130 & $10 \%$ & \multirow{3}{*}{$130 \pm 1^{\circ} \mathrm{C}$} \\
\hline YS 20-130 & YH 20-130 & $20 \%$ & \\
\hline YS $30-130$ & YH 30-130 & $30 \%$ & \\
\hline YS $10-140$ & YH $10-140$ & $10 \%$ & \multirow{3}{*}{$140 \pm 1^{\circ} \mathrm{C}$} \\
\hline YS 20-140 & YH 20-140 & $20 \%$ & \\
\hline YS 30-140 & YH 30-140 & $30 \%$ & \\
\hline
\end{tabular}

Por cada una de las mezclas se confeccionaron cinco probetas cúbicas (70,7 mm de lado) para su ensayo a compresión simple según la norma IRAM 1608:73 (IRAM 1608,1973); tres probetas prismáticas $(40 \times 40 \times 160 \mathrm{~mm})$ para su ensayo a flexocompresión conforme a la norma chilena NCh 144.Of1999 (NCh $144,1999)$, y seis probetas cilíndricas de $46,2 \mathrm{~mm}$ de diámetro por 96,4 mm de altura (relación 1:2), tres de las cuales para ser ensayadas a tracción por compresión diametral y el resto a compresión simple, aplicándose un procedimiento interno del laboratorio con el objeto de obtener correlaciones tracción/compresión, por carecerse de este tipo de datos para el yeso en la literatura.

Dada la cantidad de mezclas estudiadas se confeccionó el número mínimo de probetas establecido en las normas, lo cual no permitió un tratamiento estadístico de los resultados, sino que su objeto fue marcar tendencias. Las probetas de cada mezcla fueron elaboradas con la relación en peso agua/yeso obtenida de la correspondiente determinación de la pasta de consistencia normal (IRAM 1608:73). Luego del desmolde se curaron en cámara húmeda por 72 horas, tras lo cual se secaron en horno a $45^{\circ} \pm 1^{\circ} \mathrm{C}$ hasta obtener masa constante $( \pm$ $0,1 \%)$. Con posterioridad a ello, se les realizaron los ensayos físicos mencionados anteriormente.

La medición de las cargas aplicadas en los ensayos de compresión y flexión se realizó mediante un dinamómetro electrónico CIFIC de 10.000 kgf (precisión: 0,5 kgf). Se obtuvo la densidad aparente de cada probeta como la razón entre su masa desecada y el volumen; la porosidad fue valuada como el cociente entre el volumen de poros, determinado a partir de la diferencia entre el peso de la muestra al quitarla de la cámara de curado y su peso en condiciones secas, y el volumen aparente (Borrelli, 1999). El módulo de elasticidad se determinó a partir del registro de la flecha de las probetas prismáticas en el ensayo de flexo-tracción (Guillén, 2005), empleándose para tal fin un comparador digital SCHWYZ $(1 / 1000 \mathrm{~mm})$.

Para la caracterización fisicoquímica de los residuos de yeso se realizaron análisis tomando como base los establecidos en la norma IRAM 1608. Se determinó el contenido agua combinada (secado en estufa a 215-230 ㅇ) una vez triturados, tamizados y secados a las diferentes temperaturas indicadas. Además se realizaron determinaciones de calcio (método complejométrico) y sulfato (método gravimétrico), precisando para cada caso la fase de sulfato de calcio presente (anhidrita, hemihidrato o dihidrato).

\section{Resultados y discusión}

\section{Finura}

El ensayo de finura del yeso recuperado y molido, efectuado bajo tamizado húmedo conforme a norma IRAM 1608, satisfizo los requisitos requeridos por norma IRAM 1607 para los yesos tipo $\mathrm{A}$, la cual prescribe un pasante del $100 \%$ por el tamiz $\mathrm{N}^{\circ} 16$ (IRAM 1,18 mm), y mínimos del 95 y 70\% pasante por los tamices $\mathrm{N}^{\circ} 30$ (IRAM 0,600 mm) y $\mathrm{N}^{\circ} 100$ (IRAM 0,150 mm), respectivamente.

\section{Análisis físico-químicos}

El agua combinada de los residuos, molidos y secados a 100 , $110,120,130$ y 140 으, disminuyó en la medida que la temperatura se fue incrementando, conforme se ilustra en Figura 2 y Figura 3 . En la determinación del contenido de sulfato de calcio, se halló que en aquellos residuos secados a 100 y $110{ }^{\circ} \mathrm{C}$ el mayor porcentaje del sulfato de calcio se encuentra como hemihidrato, y un pequeño porcentaje aún se 
encuentra como dihidrato; en los que fueron secados a 120 130 y $140 \stackrel{\circ}{\circ}$, ya no hay contenido de dihidrato y se presentan moléculas de sulfato de calcio anhidro (Figura 4 y Figura 5).

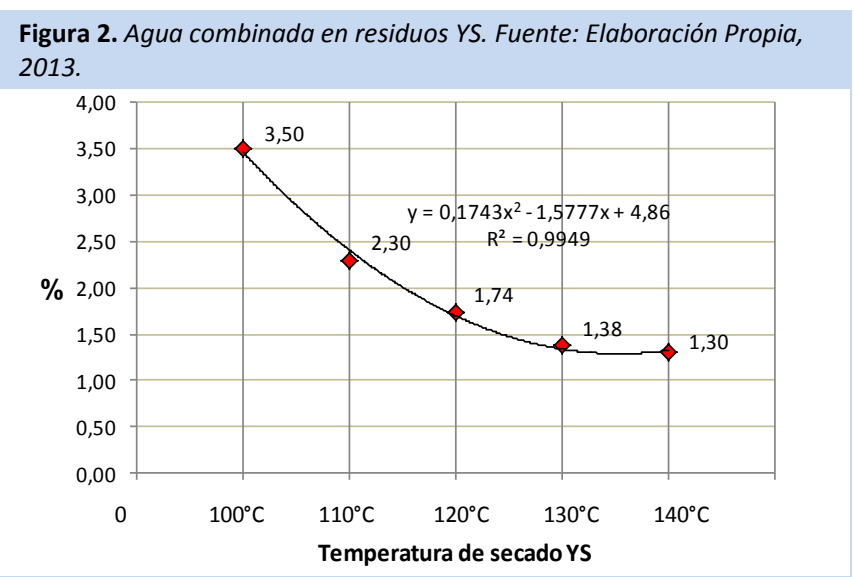

Figura 3. Agua combinada en residuos YH. Fuente: Elaboración Propia, 2013.

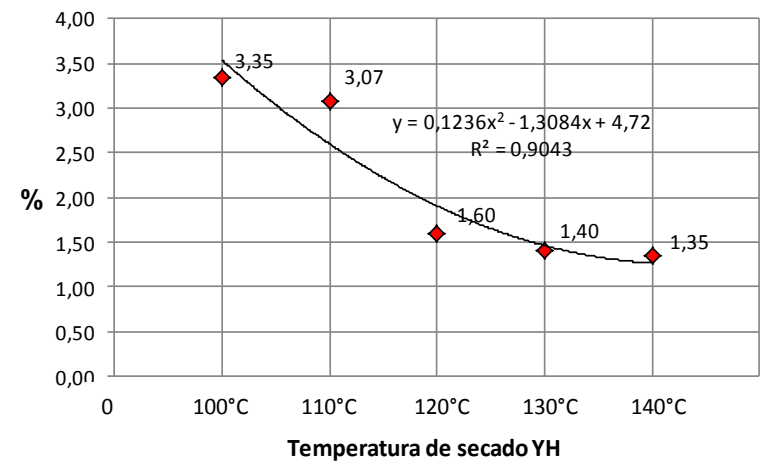

Figura 4. Contenido de los diferentes sulfatos de calcio en residuos YS. Fuente: Elaboración Propia, 2013.

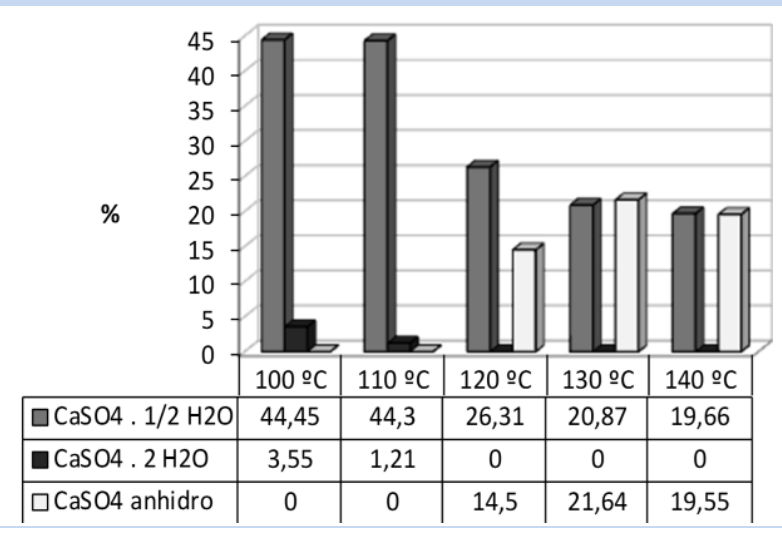

El yeso utilizado como matriz cuenta con un tenor de pureza con un contenido mínimo del $85 \%$ de $\mathrm{CaSO}_{4} \cdot 1 / 2 \mathrm{H}_{2} \mathrm{O}$ y del $8 \%$ de $\mathrm{CaSO}_{4}$ anhidro. En función de los resultados hallados en las determinaciones practicadas sobre los YS y YH, en las Tabla 2 y Tabla 3 se presenta el encuadre de las diferentes mezclas a lo requerido por las normas de ambos países. Los análisis químicos se efectuaron según la normativa argentina.
Figura 5. Contenido de los diferentes sulfatos de calcio en residuos $\mathrm{YH}$. Fuente: Elaboración Propia, 2013

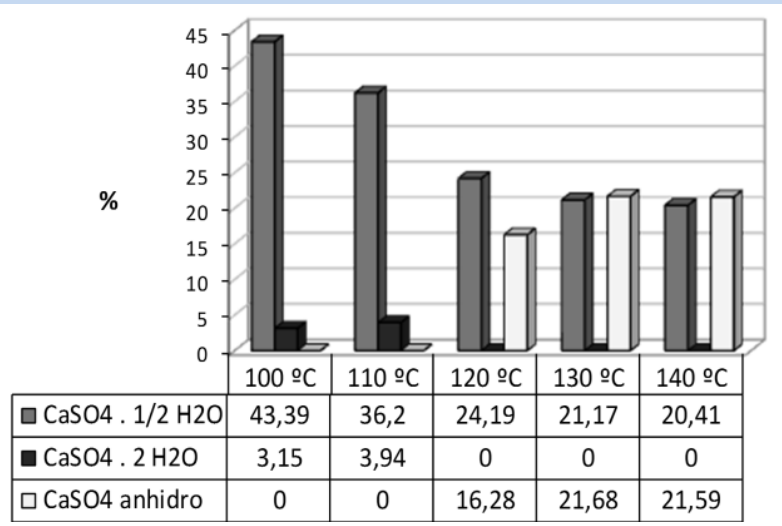

Tabla 2. Requisitos químicos. Mezclas con YS. Fuente: Elaboración Propia, 2013.

\begin{tabular}{|c|c|c|c|c|}
\hline \multirow[b]{4}{*}{ Requisito: } & \multicolumn{2}{|c|}{ IRAM 1607:70 } & \multicolumn{2}{|c|}{ NCh143.Of1999 } \\
\hline & \multicolumn{2}{|c|}{$\mathrm{CaSO}_{4} \cdot 1 / 2 \mathrm{H}_{2} \mathrm{O}+\mathrm{CaSO}_{4}$} & \multicolumn{2}{|c|}{$\mathrm{CaSO}_{4} \cdot 1 / 2 \mathrm{H}_{2} \mathrm{O}$} \\
\hline & Tipo A & Tipo B & Verifica & $\begin{array}{c}\text { No } \\
\text { verifica }\end{array}$ \\
\hline & $\geq 80 \%$ & $\geq 60 \%$ & $\geq 73 \%$ & $<73 \%$ \\
\hline YP-0 & mín.93\% & - & mín.85\% & - \\
\hline YS $10-100$ & $88 \%$ & - & $81 \%$ & - \\
\hline YS 20-100 & $83 \%$ & - & $77 \%$ & - \\
\hline YS $30-100$ & - & $78 \%$ & $73 \%$ & - \\
\hline YS 10-110 & $88 \%$ & - & $81 \%$ & - \\
\hline YS 20-110 & $83 \%$ & - & $77 \%$ & - \\
\hline YS 30-110 & - & $78 \%$ & $73 \%$ & - \\
\hline YS 10-120 & $88 \%$ & - & $79 \%$ & - \\
\hline YS 20-120 & $83 \%$ & - & $73 \%$ & - \\
\hline YS 30-120 & - & $77 \%$ & & $67 \%$ \\
\hline YS $10-130$ & $88 \%$ & - & $79 \%$ & - \\
\hline YS 20-130 & $83 \%$ & - & - & $72 \%$ \\
\hline YS 30-130 & - & $78 \%$ & - & $66 \%$ \\
\hline YS 10-140 & $88 \%$ & - & $78 \%$ & - \\
\hline YS 20-140 & $82 \%$ & - & - & $72 \%$ \\
\hline YS $30-140$ & - & $77 \%$ & - & $65 \%$ \\
\hline
\end{tabular}

Conforme se observa en ellas, quedan asegurados los requisitos químicos establecidos por la norma IRAM 1607 para yesos tipo A en todas las mezclas con hasta un $20 \%$ de residuo; las restantes se ubican dentro de lo exigido para yesos tipo B. Las evaluaciones de ajustes a la NCh143 guardan notoria similitud.

\section{Tiempos de fraguado}

Los tiempos de inicio y final de fraguado de todas las mezclas, tanto las de YS como las de YH, incluyendo la del YP, quedaron comprendidos dentro del rango 7 y 21 minutos, por lo que se ajustan a lo requerido para los yesos tipo A en Norma IRAM 1607 y la Norma NCh143. La Tabla 4 resume sus exigencias.

\section{Pasta de consistencia normal. Relaciones agua/yeso $(a / y)$}

Para el YP la relación a/y fue del 57,50\%. El rango de lo determinado para las mezclas con YS quedó comprendido entre 
el 59,50 y el $67,50 \%$, y el de las de $\mathrm{YH}$ entre el 60,75 y el $69,50 \%$. La demanda de agua ha sido creciente con el aumento del porcentaje de yeso recuperado, para cada temperatura estudiada. La relación a/y es de influencia decisiva en las características y propiedades que, finalmente, habrá de tener el yeso endurecido, tales como su densidad aparente, su porosidad y resistencia mecánica (Tesárek et al., 2011).

Tabla 3. Requisitos químicos. Mezclas con YH. Fuente: Elaboración Propia, 2013.

\begin{tabular}{|c|c|c|c|c|}
\hline \multirow[b]{4}{*}{ Requisito: } & \multicolumn{2}{|c|}{ IRAM 1607:70 } & \multicolumn{2}{|c|}{ NCh143.Of1999 } \\
\hline & \multicolumn{2}{|c|}{$\mathrm{CaSO}_{4} \cdot 1 / 2 \mathrm{H}_{2} \mathrm{O}+\mathrm{CaSO}_{4}$} & \multicolumn{2}{|c|}{$\mathrm{CaSO}_{4} \cdot 1 / 2 \mathrm{H}_{2} \mathrm{O}$} \\
\hline & Tipo A & Tipo B & Verifica & $\begin{array}{c}\text { No } \\
\text { verifica }\end{array}$ \\
\hline & $\geq 80 \%$ & $\geq 60 \%$ & $\geq 73 \%$ & $<73 \%$ \\
\hline YP-0 & mín.93\% & - & mín.85\% & - \\
\hline YH $10-100$ & $88 \%$ & - & $81 \%$ & - \\
\hline YH 20-100 & $83 \%$ & - & $77 \%$ & - \\
\hline YH 30-100 & - & $78 \%$ & $73 \%$ & - \\
\hline YH $10-110$ & $87 \%$ & - & $80 \%$ & - \\
\hline YH 20-110 & $82 \%$ & - & $75 \%$ & - \\
\hline YH 30-110 & - & $76 \%$ & - & $70 \%$ \\
\hline YH 10-120 & $88 \%$ & - & $79 \%$ & - \\
\hline YH 20-120 & $82 \%$ & - & $73 \%$ & - \\
\hline YH 30-120 & - & $77 \%$ & & $67 \%$ \\
\hline YH 10-130 & $88 \%$ & - & $79 \%$ & - \\
\hline YH 20-130 & $83 \%$ & - & - & $72 \%$ \\
\hline YH 30-130 & - & $78 \%$ & - & $66 \%$ \\
\hline YH 10-140 & $88 \%$ & - & $79 \%$ & - \\
\hline YH 20-140 & $83 \%$ & - & - & $72 \%$ \\
\hline YH 30-140 & - & $78 \%$ & - & $66 \%$ \\
\hline
\end{tabular}

Tabla 4. Tiempos de fraguado (minutos). Fuente: Elaboración Propia, 2013.

\begin{tabular}{c|c|c|c}
\hline \multirow{2}{*}{$\begin{array}{c}\text { Tiempo de } \\
\text { fraguado }\end{array}$} & \multicolumn{2}{|c|}{ IRAM 1607:70 } & \multirow{2}{*}{$\begin{array}{c}\text { NCh } \\
\text { 143.0f1999 }\end{array}$} \\
\cline { 2 - 3 } & Tipo A & Tipo B & \multirow{2}{*}{7 a 35} \\
\hline Inicio & $\geq 3$ & $\geq 3$ & \\
\hline Fin & $\leq 25$ & $\leq 35$ & \\
\hline
\end{tabular}

\section{Densidad aparente. Porosidad}

Las densidades promedio de las diferentes mezclas estudiadas fueron menores a las del YP-0 $(1,25 \mathrm{~g} / \mathrm{cm} 3)$, y tendieron a decrecer con el incremento del yeso reciclado. Dicha merma fue acompañada por el aumento de la porosidad, la cual fue del $37 \%$ para el YP-0 y se inscribió en el entorno del 38 al $42 \%$ para el YS y del 39 al $46 \%$ para el YH. En ninguno de los casos se observaron variaciones sustanciales con el incremento de la temperatura de secado.

Las Figura 6 y Figura 7 comparan las relaciones agua/yeso, las densidades y porosidades. En ellas se aprecia cómo el incremento del agua necesaria para el logro de la pasta de consistencia normal, conduce a una disminución de la compacidad por aumento de la porosidad.
Figura 6. Relaciones a/y-Densidad-Porosidad. Mezclas con YS. Fuente: Elaboración Propia, 2013.

口R.aly $\quad$ aDensidad $(\mathrm{g} / \mathrm{cm} 3) \quad$ aPorosidad

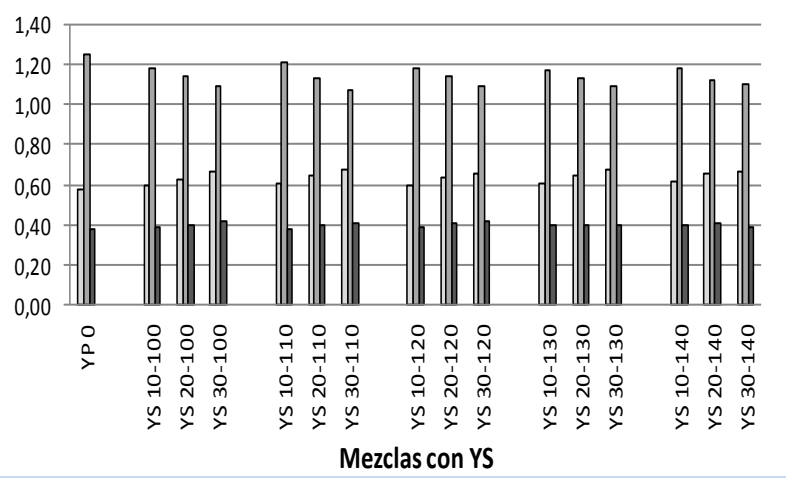

Figura 7. Relaciones $a / y$-Densidad-Porosidad. Mezclas con YH. Fuente: Elaboración Propia, 2013.

$\square R . a / y \quad \square$ Densidad $(\mathrm{g} / \mathrm{cm} 3) \quad \square$ Porosidad

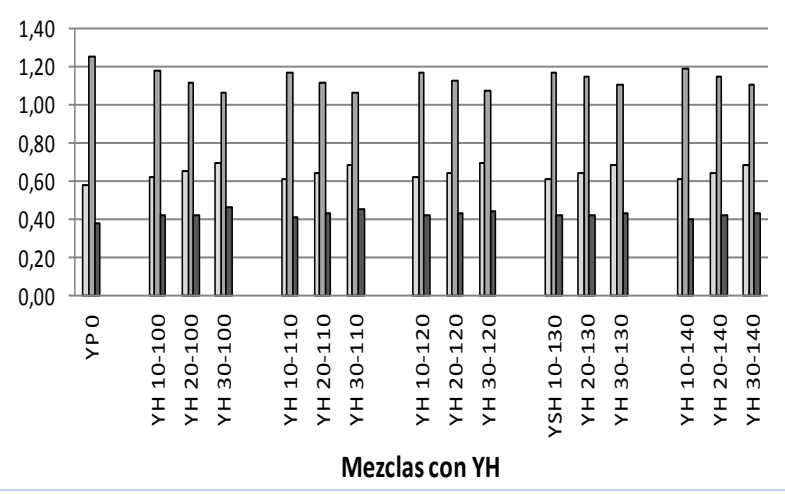

\section{Resistencia a flexo-compresión (NCh143)}

Los prismas se ensayaron a flexión y compresión conforme a la norma chilena NCh144.Of1999 de ensayos físicos para yesos calcinados. Los requisitos de aceptación están establecidos en la ya referida norma NCh143. Este ensayo no está contemplado en la norma argentina IRAM 1607 de yeso cocido para revoques. La NCh144 prescribe resistencias mínimas de 35 $\mathrm{kgf} / \mathrm{cm}^{2}$ para la flexión y de $130 \mathrm{kgf} / \mathrm{cm}^{2}$ para la compresión. Este último valor es elevado si se lo compara, particularmente, con lo requerido en la norma europea para yesos de construcción EN 13279-1:2008 (EN 13279-1, 2008). La Tabla 5 ilustra sobre lo establecido por ésta y en las normas de distintos países.

El ensayo a la flexión para las diferentes mezclas aseguró el cumplimiento de la resistencia normada para el YP y para aquellas con contenido de hasta el $10 \%$ de yeso reciclado, tanto para las de YS como YH, así como la exclusión de todas aquellas con el $30 \%$ de residuo incorporado. Solo algunas mezclas con el $20 \%$ de residuo superaron lo requerido (Figura 8 y Figura 9). Como se observa en los gráficos, algunos valores no siguen el patrón esperado de disminución de la resistencia en función del incremento del residuo. No puede afirmarse que, tanto ellos como los picos o depresiones registrados, se correspondan con datos anómalos; más bien deben atribuirse al limitado número de piezas ensayadas, aspecto este que se depuraría con el aumento de número de series de ensayos, a fin de obtener un conjunto de valores tal que permita su tratamiento estadístico descriptivo. 
Tabla 5. Requisito de resistencia a la compresión según normas de diferentes países. Fuente: Elaboración Propia, 2013.

\begin{tabular}{|c|c|c|c|c|}
\hline Norma & Designación del yeso & \multicolumn{2}{|c|}{ Resistencia a la compresión } & $\begin{array}{c}\text { Dimensiones de las } \\
\text { probetas }\end{array}$ \\
\hline $\begin{array}{l}\text { Norma Oficial Chilena } \\
\text { NCh143.Of1999 }\end{array}$ & Yeso calcinado & \multicolumn{2}{|c|}{$\geq 130 \mathrm{kgf} / \mathrm{cm}^{2}$} & $40 \times 40 \times 160 \mathrm{~mm}(1)$ \\
\hline $\begin{array}{l}\text { Norma Europea } \\
\text { EN 13279-1:2008 }\end{array}$ & $\begin{array}{l}\text { Gypsum building plaster } \\
\text { (B1) (2) }\end{array}$ & \multicolumn{2}{|c|}{$\begin{array}{l}\geq 2,0 \mathrm{~N} / \mathrm{mm}^{2} \\
\left(\cong 20 \mathrm{kgf} / \mathrm{cm}^{2}\right)\end{array}$} & $40 \times 40 \times 160 \mathrm{~mm}(1)$ \\
\hline $\begin{array}{c}\text { Norma Brasilera } \\
\text { NBR 13207:1994 (3) }\end{array}$ & $\begin{array}{c}\text { Yeso para construcción } \\
\text { civil }\end{array}$ & \multicolumn{2}{|c|}{$\begin{array}{c}>8,4 \mathrm{MPa} \\
\left(\cong 84 \mathrm{kgf} / \mathrm{cm}^{2}\right)\end{array}$} & $50 \times 50 \times 50 \mathrm{~mm}$ \\
\hline $\begin{array}{l}\text { Norma Venezolana } \\
\text { COVENIN 3638:2000 (4) }\end{array}$ & Yeso de construcción & \multicolumn{2}{|c|}{$\begin{array}{c}\geq 10.342 \mathrm{kPa} \\
\left(1500 \mathrm{lb} / \mathrm{pulg}^{2}\right) \\
\left(\cong 105 \mathrm{kgf} / \mathrm{cm}^{2}\right)\end{array}$} & $\begin{array}{c}2 \times 2 \times 2 \text { in } \\
(50,8 \times 50,8 \times 50,8 \mathrm{~mm})\end{array}$ \\
\hline \multirow{3}{*}{$\begin{array}{l}\text { Norma Argentina } \\
\text { IRAM 1607:70 }\end{array}$} & \multirow{3}{*}{$\begin{array}{l}\text { Yeso cocido para } \\
\text { revoques }\end{array}$} & Tipo A & Tipo B & \multirow{3}{*}{$70,7 \times 70,7 \times 70,7 \mathrm{~mm}$} \\
\hline & & $\geq 80 \mathrm{daN} / \mathrm{cm}^{2}$ & $\geq 60 \mathrm{daN} / \mathrm{cm}^{2}$ & \\
\hline & & \multicolumn{2}{|c|}{$\left(1 \mathrm{daN} / \mathrm{cm}^{2} \cong 1 \mathrm{kgf} / \mathrm{cm}^{2}\right)$} & \\
\hline
\end{tabular}

El ensayo a compresión se practica sobre los el par de trozos remanente del ensayo a flexión.

(2) Yeso de Construcción (B1), AENOR (UNE-EN 13279-1,2009).

(3) Associação Brasileira de Normas Técnicas (ABNT NBR 13207,1994).

(4) Fondo para la Normalización y Certificación de la Calidad (COVENIN 3638, 2000).

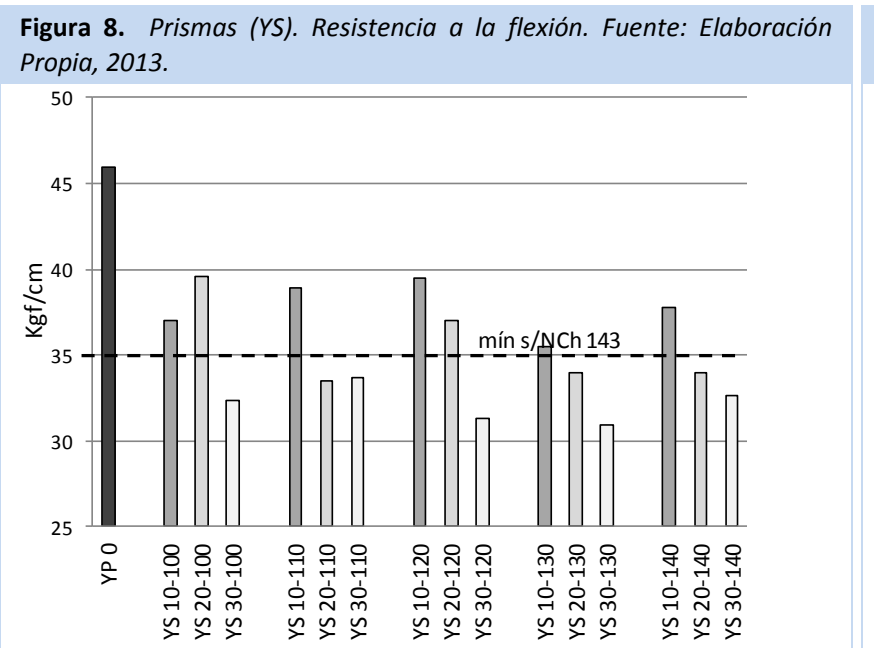

Figura 10. Prismas (YS). Resistencia a la compresión. Fuente: Autor, 2013.

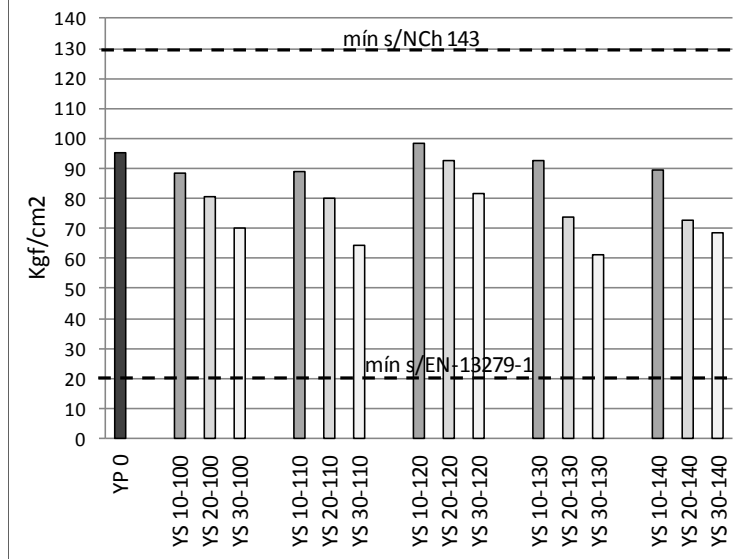

Figura 9. Prismas (YH). Resistencia a la flexión. Fuente: Elaboración Propia, 2013.

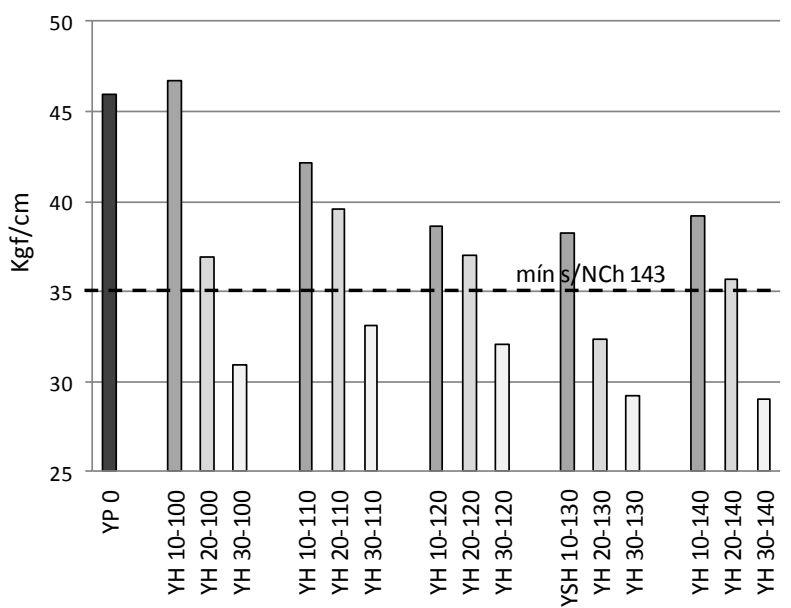

Figura 11. Prismas (YH). Resistencia a la compresión. Fuente: Elaboración Propia, 2013.

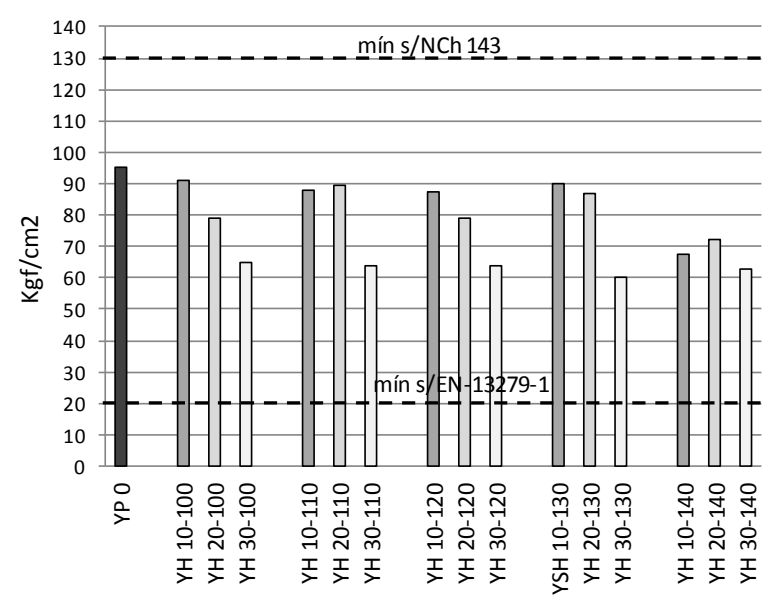


Las Figuras 10 y 11 presentan los resultados de los ensayos a compresión. Se observa que los valores alcanzados han estado por debajo de lo exigido por la norma NCh143, aun para las muestras de YP. Sin embargo, todas las mezclas cumplen con la menos exigente norma europea EN 13279-1. Las observaciones realizadas sobre los resultados de los ensayos a flexión en cuanto a las aparentes anomalías detectadas, son extensivas a este caso.

\section{Módulo E}

Los valores promedio medidos indican yesos de bajo módulo al comparárselos con lo hallado por otros investigadores (Tabla 6). Serna, Del Río, Palomo \& González (2012) informan un módulo de 824 MP para un YP-0, elaborado con una relación $a / y=0,7$.

Tabla 6. Módulo de elasticidad. Fuente: Elaboración Propia, 2013.

\begin{tabular}{c|c|c}
\hline \multicolumn{3}{|c}{ E (MPa) } \\
\hline YR (\%) & YS & YH \\
\hline $\mathbf{0 \%}$ & 230 & 230 \\
\hline $\mathbf{1 0 \%}$ & 220 & 291 \\
\hline $\mathbf{2 0 \%}$ & 230 & 231 \\
\hline $\mathbf{3 0 \%}$ & 191 & 208 \\
\hline
\end{tabular}

Tabla 7. Cilindros. Relación tracción diametral/compresión simple. Fuente: Elaboración Propia, 2013.

\begin{tabular}{c|c|c|c|c}
\hline \multicolumn{3}{c|}{ Yeso } & \multirow{2}{*}{$\begin{array}{c}\text { Hormigón } \\
\text { [28 días] (4) }\end{array}$} & $\begin{array}{c}\text { Suelo- } \\
\text { cemento } \\
\text { fluido } \\
\text { [28 días] (5) }\end{array}$ \\
$\begin{array}{c}\text { Yeso } \\
\text { Patrón } \\
\text { (YP) }\end{array}$ & $\begin{array}{c}\text { Mezclas } \\
\text { YS }\end{array}$ & $\begin{array}{c}\text { Mezclas } \\
\text { YH }\end{array}$ & & \\
\cline { 1 - 2 } $\mathbf{1 3 , 5} \%$ & $\mathbf{1 7 , 7} \%$ & $\mathbf{1 4 , 4} \%$ & & \\
\hline $\begin{array}{c}14,9 \% \\
(1,2,3)\end{array}$ & $\begin{array}{c}19,4 \% \\
(1,2)\end{array}$ & $\begin{array}{c}18,8 \% \\
(1,3)\end{array}$ & & $20-10 \%$ \\
\hline
\end{tabular}

(1) Probetas moldeadas con la incorporación de bórax al agua de amasado (1,4\% en peso); el yeso patrón fue blanco tipo $A$, con certificación IRAM.

(2) Begliardo et al. (2012a).

(3) Begliardo et al. (2012b).

(4) Tonda, Begliardo, Panigatti (2008).

(5) Sánchez, Begliardo, Casenave, Garrappa \& Denardi (2011).

\section{Resistencia a la compresión (IRAM 1607)}

Las Figura 12 y Figura 13 ilustran sobre las resistencias a la compresión de todas las mezclas con YS y YH, respectivamente. En el caso de las primeras, aquellas con hasta el $20 \%$ de yeso reciclado han superado las exigencias de la norma IRAM 1607 establecidas para yesos blancos, tipo A ( $\geq 80 \mathrm{daN} / \mathrm{cm} 2)$. Aquellas con la inclusión del $30 \%$ presentaron resistencias menores, quedando encuadradas en la categoría de yesos grises, tipo $\mathrm{B}\left(\geq 50 \mathrm{daN} / \mathrm{cm}^{2}\right)$. En cuanto a las mezclas con $\mathrm{YH}$ solo aquellas con el agregado de un $10 \%$ de reciclado superaron lo requerido para los yesos tipo A; el resto, con el $20 \%$ y el $30 \%$ de material recuperado, quedaron incluidas en la categoría de yesos tipo $B$.

En general, las menores resistencias alcanzadas por estas últimas mezclas, comparadas con sus homólogas de YS, tiene su explicación en la mayor cantidad de impurezas con las que están compuestas, dada su elaboración artesanal. Las mermas registradas en las resistencias, al pasar la composición de un 10 al $30 \%$ de reciclado, son significativas. Como en los casos anteriores, no se observan tendencias definidas en cuanto al aumento o disminución de las resistencias con el incremento de la temperatura en el rango de secado analizado.

\section{Cilindros}

El ensayo de tracción por compresión diametral aplicado a las probetas cilíndricas no está normalizado para yesos, por lo que se siguió un procedimiento interno del laboratorio similar al aplicado a probetas de hormigón, pero sin la inclusión de tablillas de reparto de carga sobre dos generatrices. Se adoptó para ello una velocidad media de carga de $20 \mathrm{~kg} / \mathrm{s}$. En los ensayos de compresión simple la velocidad media adoptada fue de $66,5 \mathrm{~kg} / \mathrm{s}$. La Tabla 7 presenta los valores promedio hallados y se los compara con registros similares obtenidos para otras mezclas con yeso reciclado y otros dos materiales de construcción, el hormigón y el suelo-cemento fluido, estos últimos ensayados a la edad de 28 días.

Figura 12. Cubos (YS). Resistencia a la compresión. Fuente: Elaboración Propia, 2013.

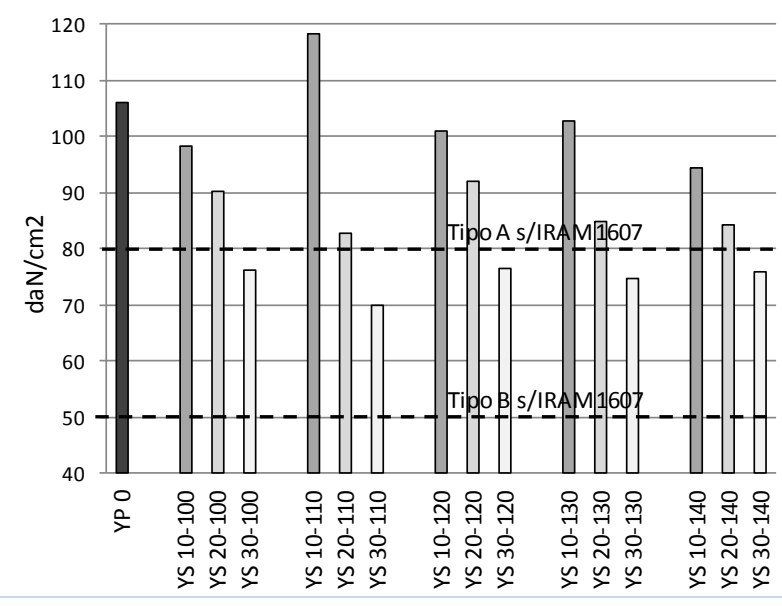

Figura 13. Cubos $(Y H)$. Resistencia a la compresión. Fuente: Elaboración Propia, 2013.

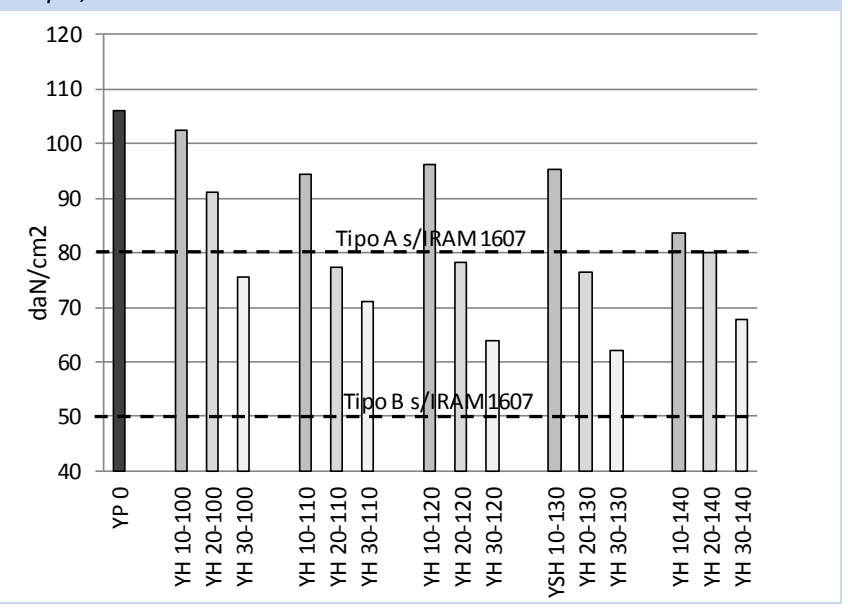




\section{Conclusiones}

La investigación realizada ha tenido por finalidad estudiar el comportamiento de mezclas de yeso recuperado de obras, bajo requisitos de aptitud establecidos en normas argentinas y chilenas, y analizar la viabilidad de su reutilización en la construcción. De lo realizado, se extraen las siguientes conclusiones: La incorporación al yeso comercial, blanco cocido para construcción, de residuos de yeso recuperados de placas laminadas, o descartes de aplicaciones en condiciones húmedas, sometidos a un proceso molienda y secado de 100 a $140^{\circ} \mathrm{C}$, en porcentajes de hasta el $30 \%$ en peso, permite obtener mezclas que conservan sus propiedades aglomerantes, con tiempos de inicio y final de fraguado enmarcados dentro de las requisitos de Norma IRAM 1607 para yesos tipo A, y los de Norma NCh143 para yesos calcinados. Las mezclas con hasta un $20 \%$ de residuo, tanto de YS como YH, cumplieron con los requisitos químicos establecidos por la norma IRAM 1607 para los yesos tipo A; aquellas con la inclusión del $30 \%$ se ubicaron dentro de lo exigido para los yesos tipo B. El ajuste a las exigencias de $\mathrm{NCh} 143$ guarda notoria similitud para con los yesos tipo $A$, en aquellas que verifican, $y$ con los yesos tipo $B$ para los que no lo hacen. La norma argentina para ensayos de yeso cocido para revoques, vigente desde principios de la década de 1970, difiere sustancialmente de la chilena para yesos calcinados utilizada para comparación, en lo que hace al tipo de probetas, ensayos físicos y requisitos de resistencia, entre otros aspectos. Por los pisos de resistencia más elevados requeridos por esta última, solo el yeso patrón y las mezclas con hasta el $10 \%$ de yeso reciclado cumplieron con la resistencia mínima a la flexión, en tanto que ninguna satisfizo la de compresión. Las resistencias a la compresión de las probetas elaboradas y ensayadas conforme a la normativa argentina, de las mezclas con hasta un $20 \%$ de yeso recuperado de aplicaciones en condiciones secas (YS), superaron las exigencias de la norma IRAM 1607 establecidas para yesos blancos tipo A, aptos para revoques finos. En el caso de las mezclas elaboradas con yeso recuperado de aplicaciones en condiciones húmedas (YH), el cumplimiento de dicho requisito solo quedó asegurado para el agregado de un $10 \%$ de reciclado. El resto de todas las mezclas que no quedaron encuadradas en esta categoría, han satisfecho con amplitud lo requerido para la los yesos grises tipo B, aptos para revoques gruesos.

En general, las resistencias alcanzadas por mezclas con yeso recogido de aplicaciones en condiciones húmedas, han sido menores que las de sus homólogas elaboradas con la incorporación de residuos de placas, debiéndose ello a su mayor contenido de impurezas.

En todos los casos, se observaron caídas en las resistencias con el incremento del porcentaje de yeso reciclado en las mezclas. No se detectaron, sin embargo, tendencias sustanciales en cuanto al aumento o disminución de las resistencias con el incremento de la temperatura de secado de los residuos en el rango de 100 a $140{ }^{\circ} \mathrm{C}$ estudiado. En este trabajo se hallaron valores que relacionan la tracción por compresión diametral con la compresión simple del yeso comercial y las diferentes mezclas, configurando un aporte novedoso por carecerse de estos datos en la literatura. Asimismo, se han provisto registros del módulo de elasticidad determinados tanto para el yeso de referencia utilizado como para las diferentes mezclas conforme al porcentaje de residuo agregado.

La investigación ha cubierto un amplio abanico de mezclas pero que, en todos los casos y por su extensión, se limitó al número mínimo de ensayos normado, con el objeto de hallar indicios de la aptitud de los residuos para su reaprovechamiento. Ello explicaría la presencia de resultados, aparentemente anómalos, en los que las resistencias de algunas mezclas han sido comparables, o incluso han superado, a las del yeso patrón. EI aumento del número de series de ensayos permitirá obtener un mayor número de valores para elaborar un tratamiento estadístico descriptivo, clarificador de tendencias y depurador de este tipo de casos.

Los estudios realizados pretenden configurar un aporte al aprovechamiento de los residuos de yeso de obras civiles, aplicados tanto en condiciones húmedas como secas, con el objeto de volver a ser utilizados con su fin original de yeso en polvo para construcción. Su reutilización redundará en los beneficios medioambientales de preservación de un recurso natural no renovable (roca de yeso) y en la reducción de consumos energéticos, además de alinearse con las nuevas legislaciones, ya vigentes en algunas provincias argentinas, sobre reducción de residuos y de "basura cero".

\section{Agradecimientos}

Este trabajo fue elaborado en el marco del PID UTN (Cód. MAUTNRA1395) "Reutilización de Residuos de Construcción con yeso en la Construcción", homologado por Rectorado de la Universidad Tecnológica Nacional. El mismo contó con la colaboración de becarios y de los Laboratorios de Ingeniería Civil y Química de la UTN F.R.Rafaela. A todos ellos, vaya nuestro agradecimiento.

\section{Referencias}

Ahmed, A., \& Ugai, K. (2011). Environmental effects on durability of soil stabilized with recycled gypsum. Cold Regions Science and Technology, 66(2-3), 84-92. doi: 10.1016/j.coldregions.2010.12.004

Ahmed, A., Ugai, K., \& Kamei, T. (2011a). Investigation of recycled gypsum in conjunction with waste plastic trays for ground improvement. Construction and Building Materials, 25(1), 208-217. doi: 10.1016/j.conbuildmat.2010.06.036

Ahmed, A., Ugai, K., \& Kamei, T. (2011b). Laboratory and field evaluation of recycled gypsum as a stabilizer agent enbankment construction. Soil and Foundations, 51(6), 975-990.[ Recuperado de https://www.jstage.jst.go.jp/article/sandf/51/6/51_6_975/_pdf_el 15.07.13]

UNE-EN 13279-1 (2009). Asociación Española de Normalización y Certificación. Yesos de construcción y conglomerantes a base de yeso para la construcción. Parte 1: Definiciones y especificaciones. Asociación Española de Normalización y Certificación: Madrid, España.

Asociación Técnica y Empresarial del Yeso. (2006). Manual de ejecución de revestimientos interiores con yeso. ATEDY (Ed.). [Recuperado de http://www.atedy.es/principal.asp el 08.03.13]

ABNT NBR 13207. (1994). Associação Brasileira de Normas Técnicas. Gesso para construção civil. Especificação. Associação Brasileira de Normas Técnicas: Río de Janeiro, Brasil.

Begliardo, H., Sánchez, M., Panigatti, M., Garrappa, S., Stellón, O., Fornari, J., \& Keller, S. (2012a). Reutilización de Residuos de Placas de Yeso. Congreso Argentina y Ambiente 2012. Mar del Plata.

Begliardo, H., Sánchez, M., Panigatti, M., Stellón, O., Garrappa, S., Fornari, J., \& Keller, S. (2012b). Caracterización del Yeso recuperado de 
Obras. VIII Congreso Internacional sobre Patología y Recuperación de Estructuras CINPAR 2012. La Plata.

Borrelli, E. (1999). ARC laboratory handbook: porosity. Rome: ICCROM. Recuperado de http://www.iccrom.org/pdf/ICCROM_14_ARCLabHandbook00_en.pdf_el 10.07.13]

EN 13279-1(2008). European Committee for Standardization. Gypsum binders and gypsum plasters - Part 1: Definitions and requirements. European Committee for Standardization: Brussels, Belgium.

Farias Nascimento, F. \& Pimentel, L. (2010). Reaproveitamento de residuo de gesso. XV Encontro de Iniciação Científica da PUC-Campinas. Campinas. [Recuperado de http://www.puccampinas.edu.br/websist/portal/pesquisa/ic/pic2010/resumos/2010924_101439_502012254_r esiaL.pdf_el 29.08.13]

COVENIN 3638 (2000). Fondo para la Normalización y Certificación de la Calidad. Yeso. Construcción y Moldeo. Requisitos. Fondo para la Normalización y Certificación de la Calidad: Caracas, Venezuela.

Gomá, F. (1979). El Cemento pórtland y otros aglomerantes. Fundamentos para la interpretación de sus comportamientos en obra. Barcelona: Editores Técnicos Asociados S.A.

González Madariaga, F. (2005). Caracterización de mezclas de residuos de poliestireno expandido (EPS) conglomerados con yeso o escayola. Su uso en la construcción. (Tesis doctoral), Universitat Politècnica de Catalunya, Barcelona. [Recuperado de http://hdl.handle.net/10803/6845 el 19.08.13]

Guillén Viñas, J. (2005). Nuevas aplicaciones de recursos yesíferos. Desarrollo, caracterización y reciclado. (Tesis doctoral), Universidad Politécnica de Madrid., Madrid. [Recuperado de http://oa.upm.es/439/1/06200503.pdf el 19.08.13]

Gypsum Recycling International (2013). Why recycle gypsum waste? [Recuperado de http://www.gypsumrecycling.biz/6688-1_Whyrecycle el 15.07.13]

IRAM 1607. (1970). Instituto Argentino de Normalización y Certificación. Yeso cocido para revoques. Características. Instituto Argentino de Normalización y Certificación: Buenos Aires, Argentina.

IRAM 1608. (1973). Instituto Argentino de Normalización y Certificación. Yeso Cocido. Métodos de ensayo generales. Instituto Argentino de Normalización y Certificación: Buenos Aires, Argentina.

NCh 143. (1999). Instituto Nacional de Normalización. Yeso calcinado. Requisitos. Instituto Nacional de Normalización: Santiago, Chile.

NCh 144. (1999). Instituto Nacional de Normalización. Yeso calcinado. Ensayos físicos. Instituto Nacional de Normalización: Santiago, Chile.

Lund-Nielsen, H. (2010, Noviembre). European long-term trends in recycled gypsum usage. Global Gypsum Magazine, 24-28. [Recuperado de http://www.globalgypsum.com/pdf/eGGNov2010nonsub.pdf el 29.08.13]

Ponce, M., \& Torres Duggam, M. (2005). Yeso. In H. Nielson \& R. Sarudiansky (Eds.), Minerales para la agricultura en latinoamérica (pp. 425-515). Buenos Aires, Argentina: UNSAM.[ Recuperado de http://www.unsam.edu.ar/publicaciones/tapas/cyted/parte5.pdf_el 02.08.13]

Sánchez, M., Begliardo, H., Casenave, S., Garrappa, S., \& Denardi, M. (2011). Componentes de suelo cemento utilizando barros de excavación para pilotes como material de baja resistencia controlada (mbrc). $11^{\circ}$ Congreso Binacional de Metalurgia y Materiales, SAM/CONAMET 2011. Rosario.

Sanz Arauz, D. (2009). Análisis del yeso empleado en revestimientos exteriores mediante técnicas geológicas. (Tesis doctoral), Universidad Politécnica de Madrid, Madrid. [Recuperado de http://oa.upm.es/1711/ el 19.08.13]
Serna, A., Del Río, M., Palomo, J., \& González, M. (2012). Improvement of gypsum plaster strain capacity by the addition of rubber particles from recycled tyres. Construction and Building Materials, 35(0). 633641. doi: 10.1016/j.conbuildmat.2012.04.093

Tesárek, P., Hájková, A. \& Plachý, T. (2011). Effect of a water-gypsum ratio on mechanical properties of gypsum. In V. Fuis (Ed.), Proceedings of 17th International Conference Engineering Mechanics 2011 (1st. ed., pp. 615-618). Svratka, Czech Republik. [Recuperado de http://engmech.cz/2011/im/im/proceedings__el 10.08.13]

Tonda, M., Begliardo, H., \& Panigatti, M. (2008). Hormigón Reciclado Sin Preselección en Origen. 17ạ Reunión Técnica de la Asociación Argentina de Tecnología del Hormigón, AATH 2008. Córdoba.

Unión Europea. (1999, Julio 16). Directiva 1999/31/CE. Diario Oficial de las Comunidades Europeas. [Recuperado de http://eurlex.europa.eu/LexUriServ/LexUriServ.do?uri=OJ:L:1999:182:0001:0019:ES:PDF el 14.08.13]

Unión Europea (2003, Enero 16). Decisión 2003/33/CE. Diario Oficial de las Comunidades Europeas. [Recuperado de http://eurlex.europa.eu/LexUriServ/LexUriServ.do?uri=OJ:L:2003:011:0027:0049:ES:PDF el 14.08.13]

U. S. Geological Survey. (2003). Mineral commodity cummaries 2003. [Recuperado de http://minerals.usgs.gov/minerals/pubs/mcs/2003/mcs2003.pdf el 02.08.13]

U. S. Geological Survey. (2013). Mineral commodity summaries 2013. [Recuperado de http://minerals.usgs.gov/minerals/pubs/mcs/2013/mcs2013.pdf el 02.08.13]

Villanueva, L. (2004). Evolución histórica de la construcción con yeso. Informes de la Construcción, 56 (493), 5-11.

Villanueva, L. (2005). Las tres edades de la construcción. Informes de la Construcción, 57 (498), 41-45. 